\title{
LETTER
}

\section{Continuous subcutaneous glucose monitoring: good enough to use in glucose regulation protocols?}

\author{
Jack JM Ligtenberg*, Margo E de Plaa and Jan G Zijlstra \\ See related research by Bridges et al., http://ccforum.com/content/14/5/R176
}

Tight glucose regulation is beneficial in subgroups of intensive care unit (ICU) patients, but may harm other subgroups. This harm may be due to hypoglycemic events. In avoiding hypoglycemia, an accurate bedside glucometry method is essential [1].

Bridges and colleagues therefore evaluated the accuracy of a continuous subcutaneous glucose monitoring system (CGMS) in critically ill children, and concluded that the system proves highly accurate [2]. We have a problem with this resolute conclusion. They report a Pearson's correlation coefficient of 0.68 , which is quite low. Vlkova and colleagues even conclude that, based on a correlation coefficient of 0.69 comparing subcutaneous glucose values and laboratory blood glucose values in 15 patients, subcutaneous devices should not be used in critically ill patients [3]. We found a correlation coefficient of 0.87 in evaluating the same CGMS in 60 critically ill patients, but were concerned with the inaccuracy in the low glucose zone: we found a difference of nearly $4 \mathrm{mmol} / \mathrm{l}$ (reference blood glucose $2.8 \mathrm{mmol} / \mathrm{l}$ versus subcutaneous sensor $6.5 \mathrm{mmol} / \mathrm{l}$ ) in one patient [4]. Bridges and colleagues report 142 subcutaneous glucose readings
$<2.2 \mathrm{mmol} / \mathrm{l}$ that were falsely low, checked against blood glucose values.

The Clarke error grid is a better way to evaluate the accuracy of a CGMS than Pearson's correlation coefficient. In most published studies, the deviation of subcutaneous measurements stays in the (wide) clinically acceptable zones of the Clarke error grid. These deviations of the CGMS system, however, when used in a tight glucose regulation protocol - and adjusting the insulin dose based on the subcutaneous readings - could have severe consequences in the individual patient, if the deviations result in an unjust rise in insulin dose. Since computerized protocols based on arterial blood samples give excellent glucose regulation with a negligible chance of hypoglycemic events [5], we decided to continue using this computerized protocol to avoid treatment-related morbidity. Subcutaneous CGMS seems not good enough in aiming for tight glucose regulation in the ICU. Intravascular CGMS, used in a closed feedback loop with insulin infusion, is promising, but has not yet been evaluated in clinical studies in critically ill patients.

\section{Authors response: Before dismissing continuous glucose monitors in ICU care, let us consider a role Mark R Rigby, Brian C Bridges, Kevin O Maher and Catherine M Preissig}

We appreciate the points raised by Dr Ligtenberg and colleagues. They suggest that because continuous glucose monitor (CGM) evaluations have not exceeded a specific Pearson's coefficient, introducing their use in ICUs is imprudent [2-4]. We believe the Pearson coefficient for our data is strong (that is, $>0.6$ ) and our Clarke error grid

*Correspondence: j.j.m.ligtenberg@icv.umcg.nl

Dept. of Critical Care, University Medical Center Groningen, Groningen, The Netherlands analysis (that is, Zone A + B >95\%) and mean absolute relative difference (15.3\%) are in line with regulatory agency approval of such devices, albeit for outpatient use [2].

Unfortunately this group does not recommend an acceptable Pearson's coefficient, how a Pearson's coefficient should be integrated with other objective assessments, or how these criteria should be modified depending on the proposed role of CGMs in ICU care. Agreed, it would be premature to use data solely from CGMs to direct insulin titrations in ICUs. As one hour or more may pass between glucose checks in many ICU glycemic 
control protocols, there may be important benefits of a technology with minute-to-minute readings that closely correlates with blood glucose levels. Adjunctive devices that continuously display surrogate, but closely correlated, patient data are not uncommon in ICUs, for example end-tidal carbon dioxide readings are often used as a proxy for arterial carbon dioxide levels and can be a vital asset in the management of mechanical ventilation. CGMs that display up-to-the minute glucose trends with alarms set at critical thresholds (incorporating leeway for inaccuracy) could be used to trigger routine blood glucose measures to guide clinical management [2].

True, we had few $(0.2 \%$ of $>64,000)$ CGM readings and no blood glucose readings of $<40 \mathrm{mg} / \mathrm{dl}(2.2 \mathrm{mmol})$, and thus cannot remark on the accuracy of CGMs in the severe hypoglycemic range. Yet we contend that if CGM devices can help maintain blood glucose levels in normoglycemic ranges they may provide critical assistance in avoiding hypoglycemia and thus their precision in low blood glucose ranges may be of less importance.

\section{Abbreviations}

CGM, continuous glucose monitor; CGMS, continuous subcutaneous glucose monitoring system; ICU, intensive care unit.

\section{Competing interests}

The authors declare that they have no competing interests.

Published: 28 January 2011

\section{References}

1. Vogelzang M, Ligtenberg JJ: Practical aspects of implementing tight glucose control in the ICU. Curr Opin Clin Nutr Metab Care 2007, 10:178-180.

2. Bridges $B C$, Preissig CM, Maher KO, Rigby MR: Continuous glucose monitors prove highly accurate in critically ill children. Crit Care 2010, 14:R176.

3. Vlkova A, Dostal P, Musil F, Smahelova A, Zadak Z, Cerny V: Blood and tissue glucose level in critically ill patients: a comparison of different methods of measuring interstitial glucose levels. Intensive Care Med 2009, 35:1318.

4. Corstjens AM, Ligtenberg JJ, Horst ICC, Spanjersberg R, Lind JSW, Tulleken J, Meertens JH, Zijlstra JG: Accuracy and feasibility of point-of-care and continuous blood glucose analyzing in critically ill ICU patients. Crit Care 2006, 10:R35.

5. Vogelzang M, Loef BG, Regtien JG, van der Horst IC, van Assen H, Zijlstra F, Nijsten MW: Computer-assisted glucose control in critically ill patients. Intensive Care Med 2008, 34:1421-1427.

doi:10.1186/cc9374

Cite this article as: Ligtenberg JJM, et al:: Continuous subcutaneous glucose monitoring: good enough to use in glucose regulation protocols? Critical Care 2011, 15:403. 